\title{
Mineralização óssea em adolescentes do sexo masculino: anos críticos para a aquisição da massa óssea
}

\author{
Bone mineralization among male adolescents: critical years for bone mass gain \\ Carla C. Silva1, Tamara B. L. Goldberg², Altamir S. Teixeira ${ }^{3}$, José C. Dalmas ${ }^{4}$
}

\section{Resumo}

Objetivo: Verificar o comportamento do conteúdo mineral ósseo e da densidade mineral óssea em adolescentes do sexo masculino em função da faixa etária e do nível maturacional dos caracteres sexuais secundários.

Métodos: 47 adolescentes saudáveis na faixa etária de 10 a 19 anos foram avaliados quanto a ingestão de cálcio, peso, estatura, índice de massa corporal, estágio puberal, densidade mineral óssea e conteúdo mineral ósseo na coluna e no fêmur proximal. A massa óssea foi mensurada através de densitometria óssea. A ingestão de cálcio foi calculada através de registro dietético de 3 dias. $O$ índice de massa corporal foi calculado pelo Índice de Quetelet, e o estágio puberal foi definido segundo os critérios de Tanner. Foi utilizada estatística descritiva, média e desvio padrão, análise de variância para comparação entre os grupos etários e teste de Tukey para localizar as diferenças significativas.

Resultados: A ingestão de cálcio não alcançou o valor mínimo de $800 \mathrm{mg}$ em várias faixas etárias estudadas. A densidade mineral óssea e o conteúdo mineral ósseo demonstraram incrementos com o avançar da idade, indicando diferenças significativas a partir dos 14 anos, bem como quando os adolescentes atingiam os estágios de maturação sexual G4. Os parâmetros de mineralização revelaram aumento pronunciado quando os adolescentes atingiam G3, porém sem significado estatístico.

Conclusão: Os resultados apontam um aumento importante na mineralização óssea durante a adolescência. A evidência de aumento na mineralização dos adolescentes foi percebida em níveis maturacionais superiores a G3. Os anos críticos para a aquisição da massa óssea neste estudo evidenciaram-se a partir dos 14-15 anos de idade.

J Pediatr (Rio J). 2004;80(6):461-7:Adolescência, eventos pubertários, caracteres sexuais secundários, massa óssea, densidade mineral óssea.

\begin{abstract}
Objective: To verify the behavior of the mineral bone content and density in male adolescents according to age and secondary sexual characters.

Methods: 47 healthy adolescents between 10 and 19 years old were assessed according to weight, height, body mass index, puberty stage, calcium intake, bone mineral density and content in the lumbar spine and in the proximal femur. The bone mass was measured through bone densitometries. The intake of calcium was calculated through a 3-day diet. The BMI (body mass index) was calculated with the Quetelet Index and the puberty stage was defined according to Tanner's criteria. The analysis used descriptive statistics such as average and standard deviation, and variance estimates to compare the different age groups. Moreover, the Tukey test was used to determine the significant differences.
\end{abstract}

Results: It was evident that the calcium intake in the different ages assessed has not reached the minimum value of $800 \mathrm{mg}$. The bone mineral density and content showed an increase after the age of 14 , as well as when the teenagers reached the sexual maturation stage G4. The mineralization parameters showed a high level when the teenagers were in the $\mathrm{G} 3$ stage, however, without statistical significance.

Conclusion: The results indicate an important level of bone mineralization during adolescence. Maturation levels superior to G3 have shown more mineralization. This study proves that the critical years for bone mass gain start after the 14-15 years old or older.

J Pediatr (Rio J). 2004;80(6):461-7: Adolescence, puberty stage, secondary sex characters, bone mass, bone mineral density.

1. Mestranda em Pediatria pelo Programa de Pós-Graduação em Pediatria, Faculdade de Medicina de Botucatu, Universidade Estadual Paulista (UNESP), São Paulo, SP. Bolsista CNPq.

2. Doutora. Professora assistente, Departamento de Pediatria, Faculdade de Medicina de Botucatu, UNESP, São Paulo, SP.

3. Professor assistente, Departamento de Doenças Tropicais e Diagnóstico por Imagem, Faculdade de Medicina de Botucatu, UNESP, São Paulo, SP.

4. Professor associado, Departamento de Matemática Aplicada, Universidade Estadual de Londrina (UEL), Londrina, PR.

Artigo submetido em 08.01.04, aceito em 25.08.04.

Como citar este artigo: Silva CC, Goldberg TBL, Teixeira AS, Dalmas JC. Mineralização óssea em adolescentes do sexo masculino: anos críticos para a aquisição da massa óssea. J Pediatr (Rio J). 2004;80:461-7. 


\section{Introdução}

A mineralização óssea tem seu início na vida fetal, estende-se por toda a infância e apresenta seu pico máximo de incremento nos anos da adolescência. Esses anos se constituem no período fundamental para a aquisição da massa óssea. Vários pesquisadores declaram o decorrer da infância e da adolescência como os períodos de maior incremento no capital mineral ósseo para ambos os sexos ${ }^{1-5}$.

Estudos relacionando a adolescência e a saúde dos ossos têm conquistado um importante espaço no cenário das pesquisas internacionais. Este fato está alicerçado no princípio cíclico que envolve a deposição da massa óssea no decorrer da trajetória da vida. O período da infância e da adolescência é marcado por uma taxa de formação óssea muito importante, com predomínio da formação sobre a reabsorção; na idade adulta, ambos os processos se estabilizam, e, a partir dos 45-50 anos, principalmente no sexo feminino, ocorre predomínio da reabsorção óssea.

A reabsorção óssea não é um processo exclusivo do sexo feminino, pois a constatação de osteopenia e osteoporose no sexo masculino tem aumentado de forma significativa ${ }^{6}$.

O pico de massa óssea contribui com aproximadamente $50 \%$ na variação do conteúdo mineral ósseo (CMO) até a idade de 65 anos $^{7}$. Nesse sentido, acredita-se que, ao se potencializar o acúmulo de massa óssea no período pubertário e a manutenção desse tecido na vida adulta, pode-se minimizar as reduções com o avançar da idade, contribuindo, assim, para a prevenção de fraturas.

Em idades mais avançadas, perda óssea em grande magnitude pode indicar a instalação de quadros osteopênicos e osteoporóticos. A osteoporose é uma desordem heterogênea, considerada um grave problema de saúde pública, que pode ser representada, em parte, por um ganho ósseo não adequado durante a infância e adolescên$\mathrm{cia}^{8}$. Fatores relacionados à nutrição, como aporte de cálcio, e aos exercícios físicos, particularmente os que envolvem maior impacto, demonstram efeitos positivos ao tecido ósseo, independentemente da fase da vida ${ }^{9}$.

O desenvolvimento, nos últimos anos, de métodos para avaliar a massa óssea com grande acurácia permitiram obter uma melhor compreensão da dinâmica do tecido ósseo. A densitometria óssea de dupla emissão com fontes de raio $X$ (DEXA) propicia uma análise altamente precisa e com baixa exposição à radiação, sendo adequada para a avaliação de crianças e adolescentes ${ }^{10-12}$.

No Brasil, poucas investigações se dedicaram a estudar a mineralização óssea em crianças e adolescentes saudáveis $^{13-16}$. Assim, o propósito deste estudo foi determinar o comportamento do CMO e da densidade mineral óssea (DMO) em adolescentes do sexo masculino em função da faixa etária (FE) e do nível maturacional dos caracteres sexuais secundários.

\section{Pacientes e métodos}

Participaram desse estudo adolescentes de 10 a 19 anos, voluntários, saudáveis e estudantes da Associação
Brasileira de Educadores Lassalistas, Colégio La Salle, da rede particular de ensino, situado no município de Botucatu (SP). A pesquisa teve aprovação da Comissão de Ética em Pesquisa da Faculdade de Medicina de Botucatu (UNESP), e os adolescentes e seus responsáveis tiveram ciência do seu conteúdo, através de explanações realizadas no ambiente escolar. Para que participassem da pesquisa, receberam e devolveram o termo de consentimento livre e esclarecido assinado pelo binômio adolescente e seu responsável.

Os critérios de inclusão exigiam que os adolescentes estivessem com peso entre os percentis 10 e 90 e estatura entre os percentis 10 e 97,5 para cada faixa de idade ${ }^{17}$, com índice de massa corporal (IMC) adequado para a idade ${ }^{18} \mathrm{e}$ com consumo diário de alimentos lácteos. Os adolescentes não poderiam ser tabagistas e/ou etilistas, não poderiam estar vinculados a qualquer modalidade esportiva extraescolar, à exceção apenas das aulas de Educação Física do próprio colégio. O controle da atividade física habitual não foi necessário, uma vez que investigações indicam que as atividades esportivas programadas são as que demonstram maior incremento na massa óssea1,2,7,9.

Como critérios de exclusão determinou-se que não participariam do estudo: os adolescentes com história de prematuridade ou baixo peso ao nascimento, os submetidos a terapia prolongada com corticóides ou que tivessem utilizado suplementação com cálcio e/ou ferro nos últimos 12 meses que antecediam a pesquisa. Também foram excluídos adolescentes que apresentassem as seguintes doenças: diabetes melito, desnutrição aguda ou crônica, doenças ósseas congênitas ou adquiridas, doenças gastrointestinais acompanhadas de má-absorção, história de nefropatia, com ou sem insuficiência renal crônica, endocrinopatias, puberdade precoce ou atrasada, consumo crônico de drogas, fibrose cística, doença celíaca ou utilização de drogas que afetam o metabolismo ósseo negativamente, como anticonvulsivantes ou antiácidos com alumínio. Quanto à avaliação dietética, foram excluídos os adolescentes que fizessem uso exclusivo de dieta vegetariana, aqueles com alto consumo de fibras, cafeína ou refrigerantes e os que não consumissem produtos lácteos diariamente.

A seqüência da coleta de dados teve início no ambiente escolar, onde, num primeiro momento, adolescentes sorteados aleatoriamente e que não apresentassem qualquer disfunção e/ou comprometimento citado nos critérios de exclusão foram convidados à mensuração do peso e da estatura. Quando a mensuração do peso e da estatura se enquadrava nos critérios propostos, os adolescentes foram questionados sobre hábito tabágico e consumo de bebidas alcoólicas. Uma vez satisfeitos esses quesitos, os adolescentes foram convidados a participar do estudo, e, uma vez voluntários, efetivou-se o contato com seus responsáveis para esclarecimentos sobre os métodos utilizados e posterior consentimento. Os adolescentes e seus responsáveis poderiam retirar o consentimento a qualquer momento do estudo sem qualquer prejuízo.

Dos 497 escolares matriculados, 47 adolescentes voluntários atenderam aos critérios de inclusão e participaram de todas as avaliações. Em função do rigor dos critérios de 
inclusão, as FE foram compostas por cinco adolescentes, à exceção da idade de 19 anos, que foi composta por dois adolescentes.

Os adolescentes voluntários que se enquadravam aos critérios de elegibilidade foram, então, convidados, junto com seus responsáveis, a comparecer ao Ambulatório de Adolescentes do Hospital das Clínicas da UNESP, onde foi realizada uma entrevista com os responsáveis, seguida de exame físico geral e especial, para que qualquer alteração física fosse detectada. Foram avaliados os caracteres sexuais secundários, e o resultado foi confrontado aos critérios de Tanner ${ }^{19}$. Para a verificação do impacto dos estágios pubertários sobre a mineralização óssea, o nível maturacional, através da inspeção visual dos genitais, foi confrontado com os resultados do CMO e da DMO obtidos pela DEXA.

$\mathrm{Na}$ seqüência, efetuou-se a caracterização dietética mediante a utilização de um registro alimentar de 3 dias, com o objetivo de obter informações sobre consumo, preferências, recusas de alimentos e realização das principais refeições relacionadas ao cálcio e possíveis fatores que pudessem interferir na biodisponibilidade deste mineral ${ }^{20}$. A quantificação centesimal dos inquéritos alimentares foi efetuada mediante a utilização de um sistema computacional de análise nutricional desenvolvido pelo Departamento de Nutrição da Faculdade de Saúde Pública da Universidade de São Paulo21.

Os exames para determinação do $\mathrm{CMO}$, expresso em gramas, e da DMO, expressa em g/ $\mathrm{cm}^{2}$, de cada adolescente foram realizados através de uma unidade de DEXA, utilizando um aparelho Hologic QDR 2000-Plus. A avaliação da massa óssea foi realizada na coluna lombar, entre $L 1$ e L4, e na região do fêmur proximal (colo femoral, trocanter, intertrocanter e triângulo de Ward).
Os dados foram armazenados e analisados no programa Statistica versão V. A FE entre 10 e 19 anos foi agrupada da seguinte forma: 10 anos completos a 11 anos, 11 meses e 29 dias (FE 1); 12 anos a 13 anos, 11 meses e 29 dias (FE 2); 14 anos a 15 anos, 11 meses e 29 dias (FE 3); 16 anos a 17 anos, 11 meses e 29 dias (FE 4); e 18 anos a 19 anos, 11 meses e 29 dias (FE 5). Na caracterização dos adolescentes, utilizou-se estatística descritiva (média e desvio padrão) para medidas de peso, estatura, IMC e média de 3 dias da ingestão de cálcio. A seguir foi realizada análise de variância para comparação entre todas as FEs e níveis maturacionais frente ao CMO e a DMO e teste de Tukey para localizar as diferenças significativas. Foi considerado um nível de significância de 5\%.

\section{Resultados}

Este estudo objetivou determinar e compreender a mineralização óssea de adolescentes brasileiros do sexo masculino. Nesse sentido, num primeiro momento, apresentam-se as características gerais da população de adolescentes estudados relativas a peso corporal, estatura e IMC (Tabela 1). Observa-se que os valores médios para esses indicadores, para cada uma das FE dos adolescentes do presente estudo, são similares aos do National Center for Health Statistics (NCHS), uma vez que, para serem individualmente incluídos, seus indicadores deveriam ser aqueles considerados na seção de métodos ${ }^{17,18}$. Observa-se aumento no peso corporal, estatura e IMC com o avançar da idade, evidenciando diferenças significantes a partir dos 14 aos 15 anos de idade (FE 3) para esses parâmetros (Tabela 1).

Tabela 1 - Caracterização dos adolescentes de acordo com as variáveis peso $(\mathrm{kg})$, estatura $(\mathrm{m}), \mathrm{IMC}\left(\mathrm{kg} / \mathrm{m}^{2}\right)$ e aporte total de cálcio ( $\mathrm{mg} / \mathrm{dia})$

\begin{tabular}{|c|c|c|c|c|}
\hline $\begin{array}{l}\text { Faixa etária } \\
\text { Idade (anos) }\end{array}$ & $\begin{array}{l}\text { Peso }(\mathrm{kg}) \\
\text { X } \pm \text { DP }\end{array}$ & $\begin{array}{c}\text { Estatura (m) } \\
\quad X \pm D P\end{array}$ & $\begin{array}{c}\mathrm{IMC}\left(\mathrm{kg} / \mathrm{m}^{2}\right) \\
X \pm D P\end{array}$ & $\begin{array}{c}\text { Cálcio (mg/dia) } \\
\text { X } \pm \text { DP }\end{array}$ \\
\hline$(F E 1) 10-11(n=10)$ & $36,25 \pm 4,54 *$ & $1,425 \pm 0,06^{\dagger}$ & $17,78 \pm 1,31$ & $783 \pm 236$ \\
\hline$(F E 2) 12-13(n=10)$ & $43,17 \pm 9,08 *$ & $1,540 \pm 0,08^{\ddagger}$ & $17,99 \pm 1,83$ & $740 \pm 198$ \\
\hline$(F E 3) 14-15(n=10)$ & $57,55 \pm 8,86^{\neq \dagger}$ & $1,694 \pm 0,07^{\neq \dagger}$ & $19,97 \pm 2,06^{\ddagger}$ & $887 \pm 228$ \\
\hline$(F E 4) 16-17(n=10)$ & $64,06 \pm 7,51^{\neq \dagger}$ & $1,729 \pm 0,07^{\ddagger \dagger}$ & $21,39 \pm 1,79^{\neq \dagger}$ & $894 \pm 275$ \\
\hline$(F E 5) 18-19(n=7)$ & $70,34 \pm 3,20^{\text {f†* }}$ & $1,803 \pm 0,05^{\neq \dagger}$ & $21,65 \pm 1,16^{\ddagger \dagger}$ & $1.073 \pm 434^{\dagger}$ \\
\hline Total $(n=47)$ & $53,24 \pm 14,37$ & $1,628 \pm 0,15$ & $19,64 \pm 2,98$ & $863 \pm 280$ \\
\hline
\end{tabular}

\footnotetext{
Análise de variância ANOVA e teste de Tukey para localização das diferenças entre as faixas etárias. Símbolos indicam diferenças entre faixas etárias $(p<0,05)$.

* Diferença existente entre FE 3 e os demais grupos.

$\dagger$ Diferença existente entre FE 2 e os demais grupos.

‡ Diferença existente entre FE 1 e os demais grupos.

$\mathrm{IMC}=$ índice de massa corporal; DP = desvio padrão.
} 
Através do preenchimento e posterior análise do recordatório alimentar, verifica-se que, entre as FE estudadas, a ingestão de cálcio variou de $740 \pm 198 \mathrm{mg} /$ dia a $1.073 \pm 434$ $\mathrm{mg} / \mathrm{dia}$, com média de $863 \pm 280 \mathrm{mg} / \mathrm{dia}$.

Na Tabela 2 estão expressos os valores do CMO e da DMO para as regiões da coluna lombar e fêmur proximal relacionados às idades dos adolescentes. O tratamento estatístico indicou diferenças significantes a partir dos 14 aos 15 anos (F3) no CMO da coluna lombar: $\mathrm{F} 3 \neq \mathrm{F} 1 \mathrm{p}=$ 0,$0003 ; F 3 \neq F 2 p=0,0352$ e $F 3 \neq F 5 p=0,0013$, e no fêmur proximal: $F 3 \neq F 1 p=0,00014$ e $F 3 \neq F 2 p=0,0043$. Quanto à $\mathrm{DMO}$ da coluna lombar, temos $\mathrm{F} 3 \neq \mathrm{F} 1 \mathrm{p}=0,0009$ e $\mathrm{F} 3 \neq$ $\mathrm{F} 5 \mathrm{p}=0,0021$, e, na região do fêmur proximal, $\mathrm{F} 3 \neq \mathrm{F} 1 \mathrm{p}=$ 0,00014 e $\mathrm{F} 3 \neq \mathrm{F} 2 \mathrm{p}=0,0043$.

Os parâmetros da mineralização óssea foram confrontados com o nível de maturação sexual, particularmente com o desenvolvimento dos genitais, com o intuito de verificar os estágios pubertários que indicassem maior incremento da massa óssea. Os resultados indicaram diferenças significantes em G4 e G5 tanto para o CMO (CMO coluna G4 $\neq$ G1, $\mathrm{G} 2$, G3 e G5 p < 0,004 e G5 $\neq \mathrm{G} 1$, G2 e G4 p < 0,0006, e CMO fêmur $\mathrm{G} 4 \neq \mathrm{G} 1$, G2 e G3 $p<0,004$; G5 $\neq \mathrm{G} 1$, G2 e G3 $p<$ 0,0006 ) como para a DMO (DMO coluna $\mathrm{G} 4 \neq \mathrm{G} 1, \mathrm{G} 2$, G3 e G5 $p<0,018 ; G 5 \neq G 1, G 2, G 3$ e G4 $p<0,0012$, e DMO fêmur $\mathrm{G} 4 \neq \mathrm{G} 1$, G2 e G3 p < 0,0107; G5 $\neq \mathrm{G} 1$, G2 e G3 $p<$ $0,001)$. Tal constatação revela que, entre $\mathrm{G} 1$ e $\mathrm{G} 3$, os parâmetros de mineralização não se alteram de forma significativa.

A Figura 1 ilustra a variação do CMO de acordo com as FE. É possível verificar um incremento crescente dos 10 aos 19 anos, com diferenças significantes entre 14 e 15 anos para o CMO do fêmur proximal e da coluna lombar, respectivamente. É possível observar que o CMO da coluna é superior ao do fêmur proximal em todas as idades.
Na Figura 2, quando se relaciona a DMO às FE, observase comportamento similar ao do CMO. A DMO demonstra diferenças significativas a partir dos 14 aos 15 anos na região da coluna lombar e no fêmur proximal; no entanto, os valores da DMO do fêmur são superiores aos encontrados na coluna.

O comportamento da mineralização óssea foi similar e crescente com o avanço da maturação sexual (Figuras 3 e 4), indicando diferenças significantes em G4 e G5 para o CMO e para a DMO em ambos locais avaliados.

Contudo, é visível, nas figuras indicadas, que, a partir de G3, já se constata um aumento pronunciado tanto para o CMO como para a DMO.

\section{Discussão}

Vários fatores são listados como sendo importantes para o acréscimo máximo de DMO durante a puberdade. Entre eles, destacam-se as contribuições de ordem genética, as alterações nas dimensões corporais, peso e estatura, 0 perfil hormonal, que conduz à maturidade esquelética e sexual, a prática de exercícios físicos pelos adolescentes e a adequada ingestão de cálcio nessa faixa de idade, que se refletem na intensa mineralização óssea6,8,22-24.

Quanto ao aporte de cálcio dietético, verifica-se que, no presente estudo, a ingestão diária dos adolescentes não alcançou as recomendações mínimas em todas as idades estudadas. A ingestão variou de $740 \mathrm{mg} / \mathrm{dia}$ a $1.073 \mathrm{mg} / \mathrm{dia}$, quando o considerado "ideal" para adolescentes tanto do sexo feminino como do masculino, de acordo com as Dietary Reference Intakes (DRI) seria de $1.300 \mathrm{mg} / \mathrm{dia}^{25}$. A literatura aponta que o valor máximo de ingestão não deveria ultrapassar $2.500 \mathrm{mg} /$ dia, valor esse que não foi atingido por qualquer adolescente de nossa amostra. Entretanto, os

Tabela 2 - Médias e desvio padrão do conteúdo mineral ósseo e da densidade mineral óssea nas regiões da coluna (L1-L4) e do fêmur proximal com relação às faixas de idade

\begin{tabular}{|c|c|c|c|c|}
\hline Idade (anos) & $\begin{array}{c}\text { CMO-Coluna } \\
\text { (gramas) }\end{array}$ & $\begin{array}{l}\text { DMO-Coluna } \\
\left(\mathrm{g} / \mathrm{cm}^{2}\right)\end{array}$ & $\begin{array}{l}\text { CMO-Fêmur } \\
\text { (gramas) }\end{array}$ & $\begin{array}{l}\text { DMO-Fêmur } \\
\left(\mathrm{g} / \mathrm{cm}^{2}\right)\end{array}$ \\
\hline (FE 1) 10-11 & $28,75 \pm 4,66^{*}$ & $0,616 \pm 0,056^{*}$ & $25,51 \pm 4,15^{*}$ & $0,781 \pm 0,08 *$ \\
\hline (FE 2) 12-13 & $37,11 \pm 11,42 *$ & $0,721 \pm 0,123$ & $33,58 \pm 11,17^{*}$ & $0,846 \pm 0,09$ \\
\hline (FE 3) 14-15 & $51,92 \pm 14,70^{\dagger \neq}$ & $0,843 \pm 0,139^{\dagger}$ & $49,67 \pm 10,29^{\dagger \neq}$ & $1,009 \pm 0,15^{\dagger}$ \\
\hline (FE 4) 16-17 & $60,98 \pm 11,35^{\dagger \neq}$ & $0,954 \pm 0,117^{\dagger \neq}$ & $56,58 \pm 11,74^{\dagger \neq}$ & $1,123 \pm 0,18^{\dagger \neq}$ \\
\hline (FE 5) 18-19 & $76,72 \pm 10,46^{\dagger \neq *}$ & $1,094 \pm 0,132^{\dagger キ} *$ & $62,15 \pm 7,35^{+\ddagger}$ & $1,205 \pm 0,11^{\dagger \neq}$ \\
\hline Total $(n=47)$ & $49,46 \pm 19,52$ & $0,830 \pm 0,197$ & $44,43 \pm 16,48$ & $0,980 \pm 0,2$ \\
\hline
\end{tabular}

\footnotetext{
Análise de variância ANOVA e teste de Tukey para localização das diferenças entre as faixas etárias. Símbolos indicam diferenças entre faixas etárias $(p<0,05)$.

* Diferença existente entre FE 3 e os demais grupos.

$\dagger$ Diferença existente entre FE 1 e os demais grupos.

‡ Diferença existente entre FE 2 e os demais grupos.

$\mathrm{CMO}=$ conteúdo mineral ósseo; $\mathrm{DMO}=$ densidade mineral óssea
} 


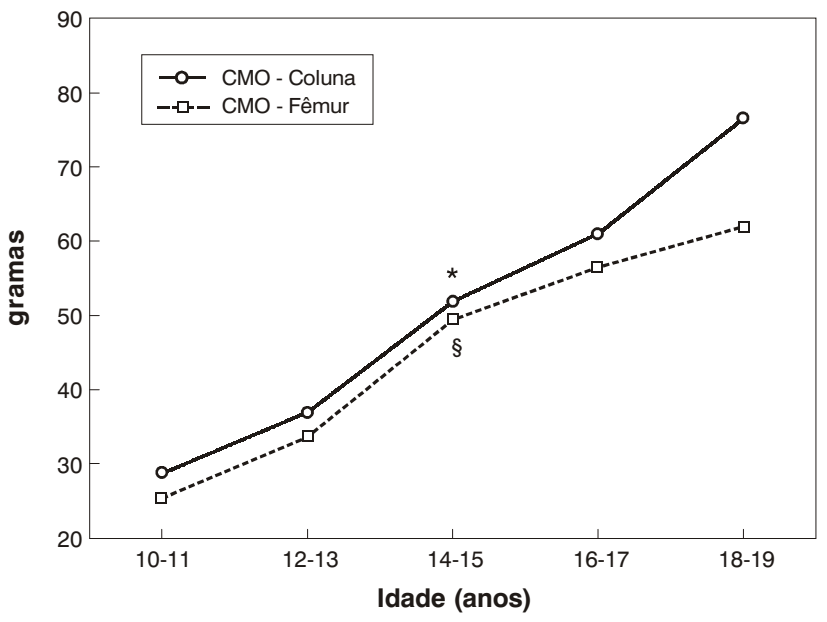

Análise de variância ANOVA e teste de Tukey para localização das diferenças entre as faixas etárias.

* Indica > significativo na CMO - Coluna $(p<0,05)$.

$\S$ Indica > significativo na CMO - Fêmur $(\mathrm{p}<0,05)$.

Figura 1 - Variação do conteúdo mineral ósseo entre as faixas etárias

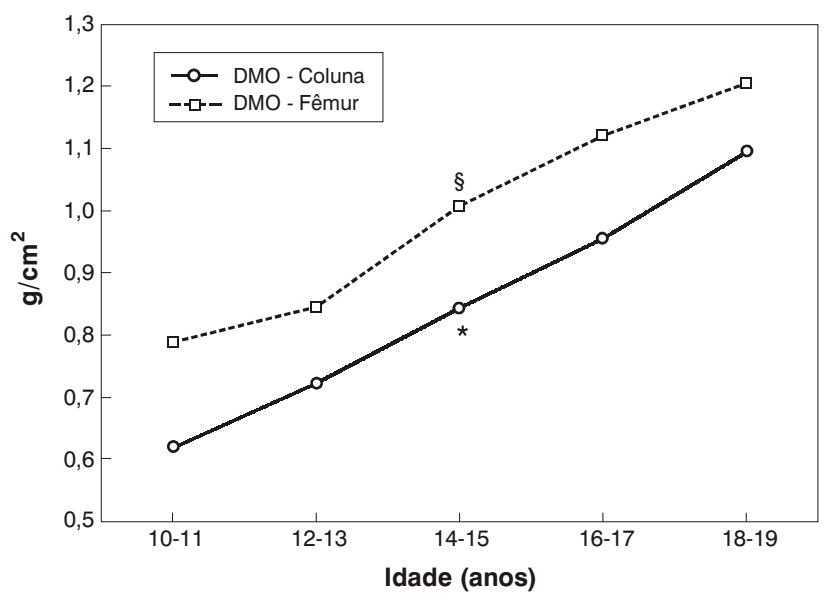

Análise de variância ANOVA e teste de Tukey para localização das diferenças entre as faixas etárias.

* Indica > significativo na DMO - Coluna $(p<0,05)$.

$\S$ Indica $>$ significativo na CMO - Fêmur $(p<0,05)$.

Figura 2 - Variação da densidade mineral óssea entre as faixas etárias

valores encontrados em nosso estudo foram superiores àqueles apresentados em outros estudos brasileiros para a mesma FE ${ }^{26-28}$. Investigações transversais realizadas com crianças e adolescentes indicam efeitos benéficos advindos da adequada ingestão de cálcio sobre o pico de massa óssea ${ }^{8}$. Parece evidente que a baixa ingestão desse mineral durante a fase de crescimento de crianças e adolescentes resulta em menor mineralização óssea quando comparada à de indivíduos da mesma FE que tiveram ingestão adequa-

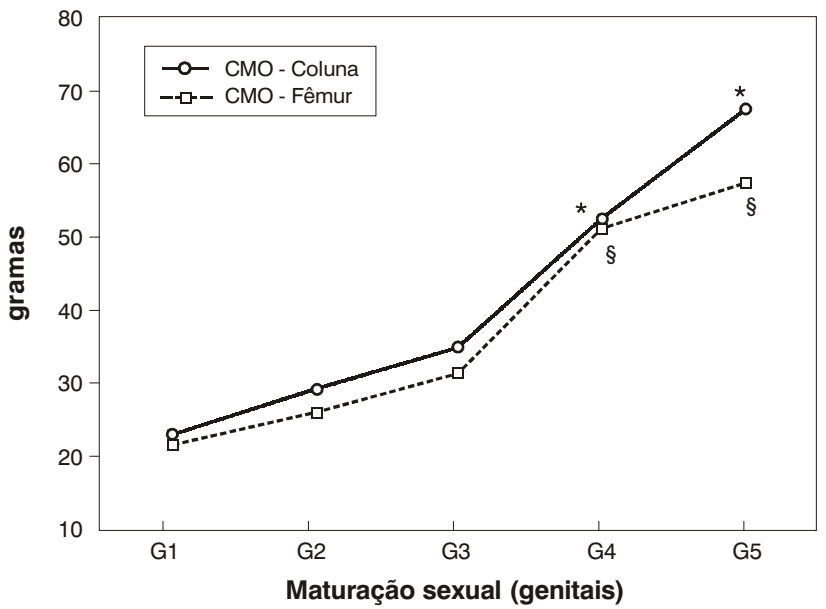

Análise de variância ANOVA e teste de Tukey para localização das diferenças entre as faixas etárias.

* Indica diferença CMO - Coluna com G1, G2 e G3.

$\S$ Indica diferença CMO - Fêmur com G1, G2 e G3.

Figura 3 - Variação do conteúdo mineral ósseo em relação aos níveis de maturação sexual

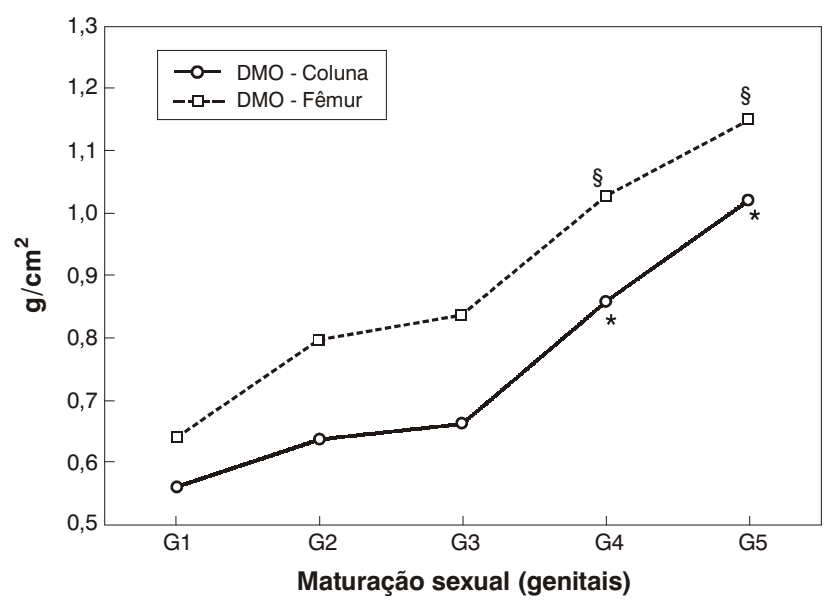

Análise de variância ANOVA e teste de Tukey para localização das diferenças entre as faixas etárias.

* Indica diferença DMO - Coluna com G1, G2 e G3.

§ Indica diferença DMO - Fêmur com G1, G2 e G3.

Figura 4 - Variação da densidade mineral óssea em relação aos níveis de maturação sexual

da de cálcio ${ }^{29}$. A comprovação do impacto benéfico da ingestão de cálcio sobre a mineralização óssea de crianças e adolescentes foi relatada em estudo longitudinal conduzido por Lee et al. ${ }^{30}$. Os pesquisadores ofereceram suplementação de carbonato de cálcio a $800 \mathrm{mg} /$ dia por 18 meses para crianças de ambos os sexos com média de idade de 8,5 anos. Os resultados demonstraram um aumento significativo no CMO da coluna lombar das crianças suplementadas em relação ao grupo controle. 
Os benefícios potenciais da dieta rica em cálcio e dos exercícios físicos efetuados sistematicamente durante a infância e adolescência são relatados por vários autores $^{2,5,31}$. Esses comportamentos formam a base de um estilo de vida saudável relacionado à massa óssea. Dados da literatura ressaltam que a incorporação de hábitos adequados em populações pediátricas tende a se perpetuar durante a vida adulta e minimiza o risco de fraturas em idades posteriores $2,8,9$.

Com relação ao dimorfismo sexual, tanto na ingestão de cálcio como nos níveis de atividade física durante a infância e a adolescência, os adolescentes revelam hábitos mais adequados quando comparados às adolescentes 8,32 .

Durante a puberdade, dois eventos ocorrem quase simultaneamente. Um fenômeno é o estirão de crescimento físico, caracterizado por incremento substancial nas medidas de estatura; o outro consistiria em atingir o pico de massa óssea. Ambas as situações, aparentemente, são mediadas por similar cascata hormonal, incluindo os marcadores bioquímicos de formação óssea, que estão relacionados fortemente ao IGF-18,33.

Os resultados do presente estudo indicam diferenças significantes a partir dos 14 aos 15 anos tanto no CMO como na DMO, nas regiões da coluna lombar e do fêmur proximal. Rubin et al. ${ }^{8}$ avaliaram a DMO de 299 crianças e adolescentes de ambos os sexos na FE de 6 a 18 anos. Os resultados indicaram aceleração importante na DMO na coluna lombar a partir dos 13 anos no sexo masculino, com estabilização nos resultados próximo dos 15 aos 16 anos, o que denota similaridade ao presente estudo. Em investigação com 207 crianças e adolescentes caucasianos de ambos os sexos entre as idades de 9 e 17 anos, observou-se diferença pronunciada no sexo masculino, tanto na coluna lombar como no fêmur proximal, entre 13 e 17 anos $^{22}$. Para Theintz et al. ${ }^{23}$, o período dos 13 aos 17 anos foi fundamental para o incremento da DMO na coluna lombar e no fêmur proximal.

Tudo indica que a FE dos 14 aos 16 anos seja um período crítico para o processo de mineralização óssea.
Esses dados estão de acordo com várias investigações onde se observou um aumento linear na massa óssea durante a infância, com incremento exponencial durante a puberdade, em vários sítios ósseos estuda$\operatorname{dos}^{8,13,14,31,34}$.

Embora a idade seja um indicador temporal muito importante no tocante às alterações que ocorrem no período da adolescência, ela é limitada quanto às constantes modificações que ocorrem no período pubertário, uma vez que níveis maturacionais diferenciados são observados para uma mesma idade.

Os resultados apresentados nas Tabelas 2 e 3 revelam que, a partir dos 14 aos 15 anos de idade e entre os níveis maturacionais G4-G5, os adolescentes do sexo masculino experimentam um acréscimo significativo na massa óssea, que reflete ganhos no CMO e na DMO nos locais pesquisados, ou seja, coluna lombar e fêmur proximal. Essa FE e este perfil maturacional indicam fases significativamente críticas na aquisição da massa óssea, com reflexos na alta taxa de mineralização.

O presente estudo evidencia o incremento da mineralização óssea durante os anos da puberdade concomitantemente ao significativo aumento nas dimensões corporais e sua relação com o amadurecimento dos caracteres sexuais secundários.

No que tange à população aqui estudada, é importante ressaltar que esses adolescentes fazem parte de um estrato diferenciado socialmente, com peso e estatura adequados e ingestão de cálcio superior à observada em vários estudos publicados no Brasil ${ }^{26-28}$. Assim, os resultados apresentados são indicativos da variação do CMO e da DMO de uma amostra de adolescentes saudáveis. Até o presente momento, ele se mostra como único referencial que tenha levado em consideração os critérios de inclusão e exclusão apresentados. No entanto, outros pesquisadores deverão ter cautela ao confrontar seus dados aos encontrados no presente estudo, uma vez que nossos dados fazem parte de uma investigação regional.

Tabela 3 - Médias e desvio padrão do conteúdo mineral ósseo e da densidade mineral óssea nas regiões da coluna e do fêmur proximal em função do nível de maturação sexual

\begin{tabular}{|c|c|c|c|c|}
\hline $\begin{array}{l}\text { Desenvolvimento } \\
\text { dos genitais }\end{array}$ & $\begin{array}{l}\text { CMO-Coluna } \\
\text { (gramas) }\end{array}$ & $\begin{array}{l}\text { DMO-Coluna } \\
\left(\mathrm{g} / \mathrm{cm}^{2}\right)\end{array}$ & $\begin{array}{l}\text { CMO-Fêmur } \\
\text { (gramas) }\end{array}$ & $\begin{array}{l}\text { DMO-Fêmur } \\
\left(\mathrm{g} / \mathrm{cm}^{2}\right)\end{array}$ \\
\hline $\mathrm{G} 1$ & $22,87 \pm 0,00$ & $0,561 \pm 0,00$ & $21,58 \pm 0,00$ & $0,643 \pm 0,000$ \\
\hline $\mathrm{G} 2$ & $29,29 \pm 4,82$ & $0,636 \pm 0,060$ & $26,02 \pm 4,22$ & $0,798 \pm 0,072$ \\
\hline G3 & $34,90 \pm 8,58$ & $0,662 \pm 0,116$ & $31,49 \pm 5,48$ & $0,837 \pm 0,061$ \\
\hline G4 & $52,51 \pm 14,24 *$ *† & $0,858 \pm 0,118 *$ *† & $51,24 \pm 14,36 *$ *† & $1,030 \pm 0,186$ *†キ \\
\hline G5 & $67,03 \pm 13,38 * t \neq$ & $1,010 \pm 0,139 *^{\dagger \ddagger}$ & $57,46 \pm 8,38 *+\neq$ & $1,134 \pm 0,150 *^{\dagger \neq}$ \\
\hline
\end{tabular}

Análise de variância ANOVA e teste de Tukey para localização das diferenças entre as faixas etárias. Símbolos indicam diferenças entre faixas etárias $(p<0,05)$.

* Diferença existente entre G1 e os demais níveis de maturação sexual.

$\dagger$ Diferença existente entre G2 e os demais níveis de maturação sexual.

‡ Diferença existente entre G3 e os demais níveis de maturação sexual.

$\mathrm{CMO}=$ conteúdo mineral ósseo $; \mathrm{DMO}=$ densidade mineral óssea . 
Obviamente, o aparente pico de massa óssea observado, reflexo do intenso anabolismo ósseo entre as FE de 14 a 15 anos desse estudo, deve ser confirmado através de estudos longitudinais em adolescentes brasileiros do sexo masculino. Entretanto, acreditamos ser de fundamental importância a divulgação desses resultados, uma vez que, quando confrontados aos dados da literatura internacional, indicaram grande similaridade e representatividade.

A delimitação de anos críticos para o acúmulo de tecido ósseo, particularmente na coluna lombar e no fêmur proximal, locais comumente afetados pela reabsorção óssea em idade posterior e tendo como conseqüência alto risco de fraturas, deve ser investigada com a perspectiva de implantação de futuros programas de prevenção baseados fundamentalmente na maximização do ganho ósseo durante as FE mais sensíveis, inseridas no período pubertário.

\section{Agradecimentos}

À Ilana Goldberg pela versão do resumo.

\section{Referências}

1. Pettersson $U$, Nordströnm $P$, Alfredson $H$, Henriksson-Larsén $K$, Lorentzon R. Effect of high impact activity on bone mass and size in adolescent female: a comparative study between two different types of sports. Calcif Tissue Int. 2000;67:207-14.

2. Lima F, Falco V, Baima J, Carazzato JG, Pereira RMR. Effect of impact load and active load on bone metabolism and body composition of adolescent athletes. Med Sci Sports Exerc. 2001;33:1318-23.

3. Jones G, Riley MD, Whiting S. Association between urinary potassium, urinary sodium, current diet, and bone density in prepuberal children. Am J Clin Nutr. 2001;73:839-44.

4. Outila TA, Kärkkäinen MUM, Lmberg-Allardt CJE. Vitamin D status affects serum parathyroid hormone concentrations during winter in female adolescents: associations with forearm bone mineral density. Am J Clin Nutr. 2001;74:206-10.

5. Crawford PB, Wang MC, Sabry ZI, Hudes M, van Loan M, Bachach LK. Adolescent diet is predictive of peak bone mass. Am J Clin Nutr. 2002;75:S356.

6. Lorentzon M, Lorentzon R, Bäckström T, Nordstöm P. Estrogen receptor gene polymorphism, but not estradiol levels, is related to bone density in health adolescents boys: a cross-sectional and longitudinal study. J Clin Endocrinol Metab. 1999;84: 4597-601.

7. Nordström PK, Thorsen K, Nodström G, Bergström E, Lorentzon R. Bone mass, muscle strength, and different body constitutional parameters in adolescent boys with a low or moderate exercise level. Bone. 1995;17:351-6.

8. Rubin K, Schirduan V, Gendreau P, Sarfarazi M, Mendola R, Daisky $G$. Predictors of axial and peripheral bone mineral density in health children and adolescents, with special attention to the role of puberty. J Pediatr. 1993;123:863-70.

9. Mackelvie KJ, Khan KM, Mckay HA. Is there a critical period for bone response to weight-bearing exercise in children and adolescents? A systematic review. Br J Sports Med. 2002;36: 250-7.

10. Bonjour JP. Peak bone mass, calcium, and protein intakes. Feed Toddlers Adolesc. 1996;37:31-43.

11. Boot AM, Bouquet J, Ridder MAJ, Krenning EP, Keizer-Schrama SMPFM. Determinants of body composition measures by dualenergy $\mathrm{x}$-ray absorptiometry in Dutch children and adolescents. Am J Clin Nutr. 1997;66:232-8.

12. Plapler PG. Osteoporose e exercícios. Rev Hosp Clin Fac Med Univ São Paulo. 1997;52:163-70.

13. Fonseca ASM. Densidade mineral óssea da coluna lombar com densitometria de dupla emissão com fontes de raio-x (DEXA) em crianças de 6 a 14 anos de idade [dissertação]. São Paulo: Universidade Federal de São Paulo; 1992.
14. Brandão CMA. Avaliação da densidade mineral óssea durante a puberdade, em crianças normais de São Paulo. Influência de fatores antropométricos, composição corporal e do SDHEA na massa óssea [tese]. São Paulo: Universidade Federal de São Paulo; 1999.

15. Silva CC, Teixeira AS, Goldberg TBL. Densidade e conteúdo mineral ósseo de adolescentes na faixa etária de 10 a 20 anos. Processo CNPq no130043/2003. Botucatu: Departamento de Pediatria; 2001.

16. Silva CC, Teixeira AS, Goldberg TBL. Impacto da ingestão de cálcio sobre a mineralização óssea em adolescentes. Rev Nutr PUCCAMP. 2004;17(3).

17. Hamill PVV, Drizd TA, Johnson CL, Reed RB, Roche AF, Moore WM. Physical growth: National Center for Health Statistics Percentiles. Am J Clin Nutr. 1979;32:607-29.

18. Prevalence of overweight among adolescents - United States, 1988-91. MMWR Morb Wkly Rep. 1994;43:818-21.

19. Marshall WA, Tanner JM. Variation in the pattern of pubertal changes in boy. Arch Dis Child. 1970;45:13.

20. Cintra IP, von der Heydy MED, Schitz BAS, Francheschini SCC, Taddei JAAC, Sigulen DM. Métodos de inquéritos dietéticos. Cad Nutr. 1997;13:11-23.

21. Philiphi ST, Szarfarc SC, Lattrza AR. Virtual Nutri. Versão 1.0 for Windows. Departamento de Nutrição: Faculdade de Saúde Pública Universidade de São Paulo; 1996.

22. Bonjour JP, Theintz G, Buchs B, Sloman D, Rizzoli R. Critical years and stages of puberty for spinal and femoral bone mass accumulation during adolescence. J Clin Endocrinol Metab. $1991 ; 73: 555-63$.

23. Theintz G, Buchs B, Rizzoli R, Sloman D, Clavien H, Sizonenko PC, et al. Longitudinal monitoring of bone mass accumulation in health adolescents: evidence for marked reduction after 16 years of age at the levels of lumbar spine and femoral neck in female subjects. J Clin Endocrinol Metab. 1992;75:1060-5.

24. Blanchet $C$, Giguère $Y$, Prud'homme $D$, Dumont $M$, Rousseau $F$, Dodin S. Association of physical activity and bone: influence of vitamin D receptor genotype. Med Sci Sport Exerc. 2002;34: 24-31.

25. Institute of Medicine (US). Dietary references intakes for calcium, phosphorus, magnesium, vitamin $\mathrm{D}$ and fluoride. Washington (DC): National Academy Press; 1998.

26. Albuquerque MFM, Monteiro AM. Ingestão de alimentos e adequação de nutrientes no final da infância. Rev Nutr PUCCAMP. 2002;15:291-9.

27. Garcia GCB, Gambardella AMD, Frutuoso MFP. Estado nutricional e consumo alimentar de adolescentes de um centro de juventude da cidade de São Paulo. Rev Nutr PUCCAMP. 2003;16:41-50.

28. Vargas DM, Rigotti T, Gütz CNRM, Lobe MCS, Fernandes JA. Mineralização óssea em crianças e adolescentes com diabetes melito tipo 1. J Pediatr (Rio J). 2003;79:253-8.

29. Jackman LA, Millane SS, Martin BR, Wood OB, McCabe GP, Peacock $M$, et al. Calcium retention in relation to calcium intake and postmenarcheal age in adolescent females. Am J Clin Nutr. 1997;66:327-33.

30. Lee WTK, Leung SSF, Leung DMY, Cheng JCY. A follow-up study on the effects of calcium-supplement withdrawal and puberty on bone acquisition of children. Am J Clin Nutr. 1996;64:71-7.

31. Nordström P, Pettersson U, Lorentzon R. Type of physical activity, muscle strength, and pubertal stage as determinants of bone mineral density and bone area in adolescent boys. J Bone Miner Res. 1998;13:1141-8.

32. Silva CC, Guedes JERP. Dimorfismo sexual na composição corporal e no desempenho motor de adolescentes de 10-16 anos. Rev Bras Ativ Fís Saúde. 2003;8:20-9.

33. Carrascosa A, Guissinyé M. Crescimento e mineralização do esqueleto durante a puberdade e adolescência: regulação nutricional e hormonal. An Nestlé. 1998;55:9-17.

34. Hui SL, Dimeglio LA, Longcope C, Peacock M, McClintock R, Perkins $A J$, et al. Difference in bone mass between black and white American children: attributable to body build, sex hormone levels, or bone turnover? J Clin Endocrinol Metab. 2003;88:642-9.

Correspondência:

Tamara Beres Lederer Goldberg

Departamento de Pediatria - Disciplina de Medicina do Adolescente CEP 18607-918 - Botucatu, SP

Fones: (14) 3811.6274 / 3811.6083

E-mail: tamara@fmb.unesp.br 